\title{
SILVA PARENS \\ ZUR ALLEGORISCHEN TECHNIK DES BERNARDUS SILVESTRIS
}

In der Formgeschichte der Allegorie nimmt die Cosmographia des Bernardus Silvestris eine Sonderstellung ein. Dieser Bernardus hat seit der Editio princeps seines Hauptwerkes' die typischen Phasen philologischer Forschungsgeschichte durchlaufen: Nachdem die vordringlichsten Fragen wie Herkunft, Lebenszeit und Identiät seit der Arbeit Pooles grundsätzlich geklärt sind, ${ }^{2}$ dominierte in den Arbeiten Gilsons und Curtius' sowie der vermittelnden Position Silversteins die Frage, ob die Cosmographia etwa als neopaganer, in diesem Fall platonisierender, Schöpfungsmythos zu deuten sei. ${ }^{3}$ Die Antwort fand sich einfach, indem man Bernardus auf dem Hintergrund der chartrensischen Platoniker interpretierte, eine Position, die die Unterscheidung von neuplatonischem Paganismus und orthodoxem Christentum überflüssig macht. ${ }^{4}$ Diese Diskussion um die religiösen Implikationen der Cosmographia dauert an. ${ }^{5}$ In neuerer Zeit interessiert man sich daneben vor allem für die allegorische Technik des kosmographischen Kunstmythos und seinen hermeneutischen Umgang mit der älteren Literatur. ${ }^{6}$ Die

1 C.S. Barach, J. Wrobel (edd.), De Mundi Universitate (Cosmographia), Innsbruck 1876.

2 R.L. Poole, The Masters of the Schools of Paris and Chartres in John's of Salisbury's Time, EHR 35, 1920, 326-331 beendete die von B. Hauréau, Mémoire sur quelques Chanceliers de l'Eglise de Chartres, Mémoires de l'Académie des Inscriptions et Belles Lettres 31, 1884, 86-88 und C.-V. Langlois, Maître Bemard, Bibliothèque de l'Ecole de Chartres 54, 1898, 225-250 geführte Diskussion um die Identität Bemard de Chartres' und Bemardus Silvestris' (zitiert nach W.W. Wetherbee [transl. comm.], The Cosmographia of Bemardus Silvestris, New York / London ${ }^{2} 1990$ (1973), 134, Anm. 83). Seit Poole hält man beide für nichtidentisch.

3 P. Gilson, La Cosmogonie de Bemardus Silvestris, AHDLMA 3, 1928, 5-24, E.R. Curtius, Zur Literarästhetik des Mittelalters, ZRPh 58, 1938, 129-232, T. Silverstein, The Fabulous Cosmogony of Bemardus Silvestris, MPh 46, 1948 / 49, 92-116.

4 T. Gregory, Anima mundi. La Filosofia di Guglielmo di Conches e la Scuola di Chartres, Firenze 1955, ders., Platonismo medievale, Studi e Ricerche, Roma 1958, E. Garin, Studi sul Platonismo medievale, Firenze 1958.

5 Neuerdings versucht Ch. Ratkowitsch, Die Kosmographia des Bemardus Silvestris. Eine Theodizee, Ordo 6, Köln / Weimar / Wien 1995, 10ff., 15ff. und passim ausgehend von den 'pessimistischen' Aussagen der Cosmographia eine Gesamtinterpretation des Werks als einer Theodizee aufzubauen.

6 H. Brinkmann, Mittelalterliche Hermeneutik, Tübingen 1980, P. Dronke, Fabula. Explorations into the Uses of Myth in Medieval Platonism, Leiden / Köln 1974, G.D. Economou, The Goddess Natura in Medieval Literature, Cambridge 1972, J. Whitman, Allegory. The Dynamics of an Ancient and Medieval Technique, Oxford 1987. Einen ausführlicheren Forschungsbericht liefert Ratkowitsch (wie Anm. 5), 7-14. Jüngst stellte B. Pabst, Prosimetrum. 
folgenden Seiten knüpfen an diese Richtung an und fragen nach den konkreten Einzelheiten der Technik, mit der Bernardus ein philosophisches Konzept, silva, zu einer Person, eben der Silva der Cosmographia, gemacht hat.

Wenn man den Handlungsverlauf der prosimetrischen Cosmographia betrachtet, so ergibt sich im Verhältnis zur Textquantität ein auffallendes Ungleichgewicht der dramatischen Bewegung: Jenseits von Raum und Zeit bittet Natura ihre Mutter Noys darum, dem unerträglichen Zustand Silvas abzuhelfen, die sich nicht zu einer Schöpfung artikulieren könne. Diese Struktur bietet die erste kreative Claudian-Anlehnung des Mittelalters. ${ }^{7}$. Um ihrer Bitte Nachdruck zu verleihen, schildert Natura das indecorum des vorliegenden Zustands. Die reine Materie, Silva, liege brach, die Welt, Mundus, wolle geschaffen werden. Soweit das Geschehen des ersten Metrums. Vielleicht ist es angebracht, dessen wichtigste Passagen hier auszuschreiben: ${ }^{8}$

Congeries informis adhuc, cum Silva teneret

Sub veteri confusa globo primordia rerum,

Visa deo Natura queri, mentemque profundam

Compellase Noym: ,[...]

7 Si sensu fortasse meo maiora capesso-

Mollius excudi Silvam, positoque veterno,

Posse superduci melioris imagine forme -

Huic operi nisi consentis, concepta relinquo. [...]

Silva rigens, informe chaos, concretio pugnax,

Discolor Usie vultus, sibi dissona massa,

Turbida temperiem, formam rudis, hispida cultum

Tradition und Wandel einer Literaturform zwischen Spätantike und Spätmittelalter, Ordo 4, Köln I Weimar / Wien 1994, 451-476 die formale Anlehnung der Cosmographia an Martianus Capella deutlich heraus.

7 Vgl. die Klage der Natura Claudians vor der Götterversammlung darüber, daß der Mensch so unzivilisiert dahinlebe (De Raptu Proserpinae 3, 33ff.). Als Abhilfe läßt der Göttervater Ceres auf der Suche nach ihrer Tochter die Welt durchwandern, der er so den Getreideanbau und damit die Zivilisation bringt. Über die Motivparallele hinaus, auf die zuerst Curtius (wie Anm. 3), 185 aufmerksam gemacht hat, verweist Bernardus in I 15 mit wörtlichem Zitat von De Raptu Proserpinae 3, 27f. auf sein Vorbild. Auch die Natura in Claudians De Consulatu Stilichonis 2, 424ff. erinnert an die des Bernardus. Wie in vielen anderen Zügen (Vgl. dazu Ratkowitsch [wie Anm. 5], 8, 130ff. und M.T. Donati, Metafisica, Fisica e Astrologia nel XII Secolo. Bernardo Silvestre e l'Introduzione 'Qui celum' dell' 'Experimentarius', SM 3a s. 31, 1990, 649-703, hier: 664 mit Anm. 44) ist Bernardus also vielleicht auch hier ein Vorläufer des Alanus, dessen Anticlaudianus seiner gesamten Konzeption nach intertextuell auf Claudians In Rufinum bezogen ist. In dieselbe Motivtradition gehört auch Dracontius' De Laudibus Dei 1, 332. Vergleichbares gilt für die Rezeption der Tragödien Senecas: Dazu O. Zwierlein, Spuren der Tragödien Senecas bei Bernardus Silvestris, Petrus Pictor und Marbod von Rennes, MLJ 22, 1987, 171-196, hier: $178 f$.

8 Der Text ist der Ausgabe P. Dronkes entnommen (Bernardus Silvestris, Cosmographia, Leiden 1978), der im Gegensatz zu der Ausgabe Barach-Wrobels dem neuerdings allgemein als besten anerkannten Textzeugen L folgt (Oxford Laud. miss. 515, s. xiii in.), neben dem er sieben weitere Handschriften vergleicht und die aus der bislang einzigen, leider aber immer noch nicht erschienenen kritischen Ausgabe A. Vernets (Bernardus Silvestris: Recherches sur l'Auteur et l'Euvre suivies d'une Édition critique de la Cosmographia, Diss. Paris 1938) übernommenen Lesarten B. Stocks (Myth and Science in the Twelfth Century. A Study of Bernard Silvester, Princeton / New Jersey 1972). 
Optat, et a veteri cupiens exire tumultu, Artifices numeros et musica vincla requirit. [...]

Ante pedes assistit Yle cum prole suorum, Invidiam factura tibi, quod cana capillos Informi squalore suum deduxerat evum. Rursus et ecce cupit res antiquissima nasci Ortu Silva novo, circumscribique figuris. [...] Omnibus hiis quia Silva caret, vix nomine vero Divinum censetur opus, sed lubrica cece Machina Fortune, melioribus orba patronis. [...] Ut quid ab eterno comitata Carentia Silvam?

Ornatu specieque superveniente recedat! Adde manum, rescinde globum, partesque resigna Et distingue locis: melius distincta placebunt. [...]

Der weitere Verlauf der Cosmographia ergibt sich zwanglos aus dieser Eingangssituation: In II $1^{9}$ nimmt Noys die Bitte Naturas wohlwollend an und gibt das Versprechen, sie zu erfüllen. Die Schilderung der Erfüllung bildet die Cosmographia, die also eigentlich einen Schöpfungsbericht darstellt und mannigfache Anklänge an die gängige Hexameralliteratur aufweist. ${ }^{10}$ In der Reihenfolge der geschaffenen Dinge ähnelt das Werk des Bernardus der Genesis, deren entscheidendes Prinzip, das der Diakrisis-Kosmogonie, spielt gerade im Appell der Natura an Noys eine gewichtige Rolle. ${ }^{11}$ Nur aus dieser Perspektive ergibt der eigenartige Titel 'Cosmographia' überhaupt einen Sinn. ${ }^{12}$ Allegorische Exegese ist neben der interpretatio litteralis et historica die gängige Technik der spätantiken und mittelalterlichen Genesis-Deutung. ${ }^{13}$ Die Bitten Naturae an Noys, Silva doch zu Gestalt zu verhelfen, führen also zu einer Kosmogonie sowohl des Mega- wie des Microcosmus. Der letztere bildet den zweiten Teil der Cosmographia und ist auschließlich der Erschaffung und Ausstattung des Menschen gewidmet. Skurrilerweise bildet den krönenden Endpunkt der Erschaffung des Menschen die

9 Fortan zitiere ich die Cosmographia in der Ausgabe Dronkes (wie Anm. 8) und zwar den Megacosmus in großen römischen Ziffern, den Microcosmus in kleinen, die Metra dazu in arabischen Ziffern nach Versen, die Prosa ebenso nach den Abschnitten Dronkes.

${ }_{10}$ Vgl. dazu Gilson (wie Anm. 3), passim und Whitman (wie Anm. 6), 220-222, der treffend zusammenfaßt: „The activities attributed by the Bible to a quite literal God Bernard distributes among various abstract powers who seem to act on their own" (222).

${ }^{11}$ In III scheint der Reihenfolge nach der erste Schöpfungsbericht der Genesis leicht modifiziert durch: Himmelssphäre und Gestirne (1-154), Erde und Lebewesen. Zum Trennungsprinzip einer indistinkten Mischung vgl. I $60 \mathrm{f}$.: rescinde globum partesque resigna / et distingue locis.

${ }^{12}$ Es wird zuwenig beachtet, daß Isidor in seinen Etymologiae im Zuge seiner Besprechung des Bibelaufbaus für den Pentateuch die Bezeichnung cosmographia verwendet $(6,2,1)$ : primus Moyses divinae historiae cosmographiam in quinque voluminibus edidit quod Pentatichum mominatur. Einerseits spricht dieser Befund gegen den alten Titel De Universitate Mundi, andererseits für Gilsons Theorie, die Cosmographia als allegorische Genesis-Deutung aufzufassen, und damit gegen Curtius.

13 Vgl. z. B Hugo de St. Victore, De Scripturis et Scriptoribus sacris, Migne PL 175, 13. 
Konstruktion seiner Genitalien: militans adversus Lachesim mentula (xiv 153ff.). ${ }^{14}$ Micro- und Megacosmus sind parallel gestaltet: Im ersten Teil ist das Brachliegen Silvas der Mangel, dem durch die Schöpfung abgeholfen wird, im zweiten fehlt der Schöpfung ihre Krone, der Mensch. ${ }^{15}$ Genaugenommen ist also die Struktur des ersten Metrums handlungsmotivierend auch noch für den Microcosmus. Der Bedeutung für das Ganze der Cosmographia entsprechend muß das Personal des ersten Metrums also besonders sorgfältig gestaltet, die Zeichnung der auftretenden Charaktere besonders eindringlich sein. Schon jetzt kann man annehmen, daß Bernardus hier sein gesamtes literarisches Wissen aufgeboten hat, um glaubwürdige Charaktere auf die Bühne seiner Weltentstehung zu bringen. Dieses literarische Wissen und das Talent des Bernardus, aus tradierten und ganz disparaten Einzelzügen neue Gestalten zu schaffen, zeigt die Silva-Allegorie besonders gut.

Wir reden hier ständig von 'Allegorie' - wie hätte Bernardus selbst seine Figuren wohl bezeichnet? Denn mögen auch Krayers Worte über die Allegorie in der Substanz zutreffen, ${ }^{16}$ der Terminus selbst faßt synchron das Phänomen nicht. Wie Dronke treffend festgestellt hat, handelt es sich ja bei Silva, Noys, Natura und den anderen in der Cosmographia auftretenden Gestalten nicht um einfache personifizierte Abstraktionen, sondern um komplexe, fast belebt agierende Charaktere. ${ }^{17}$ Im Unterschied zu den herkömmlichen Konzept-Allegorien (etwa in der Psychomachia des Prudentius) seien Natura und ihre Gefährtinnen ,conceptually unpredictable“. ${ }^{18}$ Die üblichen Allegorien entspringen letztlich dem Bedürfnis des Rezipienten nach Verlebendigung und Personalisierung abstrakter Konzepte, also nach deren Anschaulichkeit. In unserem Zusammenhang aber dienen die auftretenden Figuren der Zusammenfassung von proprie sprachlich nicht zu fassenden Vorgängen, die deshalb - wie schon im platonischen Timaios (28 C 2) - sub pio figmentorum velamine dargestellt werden müssen. ${ }^{19}$ Raby bestimmt diese Unfähigkeit, die Dinge beim Namen zu nennen, als typisches Merkmal präaristotelischer Naturwissenschaft. ${ }^{20}$ Der dem Bernardus Silvestris

${ }^{14}$ Von der "Tragik des Menschengeschlechts" ist in dieser hymnischen Passage pace Ratkowitsch (wie Anm. 5), 116f. wenig zu spüren. Vgl. auch J. Cohen, Be Fertile and Increase, Fill the Earth and Master It. The Ancient and Medieval Career of a Biblical Text, Ithaca / London 1989, 281-83 mit Anm. 31, der hier ,medieval rabbinic association[s]" feststellt.

15 Andere Parallelen führt Donati (wie Amn. 7), 686 mit Anm 117f. an.

${ }^{16}$ R. Krayer, Frauenlob und Natur-Allegorese, Heidelberg 1960, 7: „Die ursprüngliche Allegorie ist Personifikation und als solche eine Ausdrucksform religiösen oder betrachtenden Welterfassens, die aus der Verbindung von Poesie und Mythos hervorgeht."

17 Dronke (wie Anm. 8), 56-57.

${ }^{18}$ P. Dronke, Bernard Silvestris, Natura, and Personification, JWCI 43, 1983, 16-32, hier: 16, K. Smolak, Mythologie der Befreiung, WHB 33, 1992, 26-46, hier: 44: ,auffällig autonom“.

19 Vgl. Macrobius' Commentarius (ed. Willis), 1, 2, 1; 13; 14.

${ }^{20}$ F.J.E. Raby, Nuda Natura and Twelfth-Century Cosmology, Speculum 43, 1968, 72-77, hier: 77: „It was the arrival of the new Aristotle, with translations of the Metaphysics and Physics, and the commentary of Averroes, which offerred to the Latin West a completely naturalistic 
zugeschriebene Kommentar zu den ersten beiden Büchern De Nuptiis Mercurii et Philologiae des Martianus Capella unterscheidet zwischen allegoria = oratio sub historica narratione verum et ab exteriori diversum involvens intellectum und integumentum $=$ oratio sub fabulosa narratione verum claudens intellectum. ${ }^{21} \mathrm{Nach}$ dieser Bestimmung kommt die Bezeichnung allegoria abweichend vom heutigen Sprachgebrauch nur biblischen Handlungen zu, die hinter einer historisch wahren Begebenheit eine höhere Wahrheit offenbaren. Für die Handlung der Cosmographia ist also diese Bezeichnung abzulehnen, da Bernardus die Fiktionalität seiner buchstäblichen Handlung kaum bestritten haben wird. ${ }^{22}$ Der ebenfalls unserem Bernardus zugeschriebene Commentarius super sex libros Eneidos ${ }^{23}$ erwähnt den Unterschied zur allegoria nicht, sondern bestimmt nur den Begriff des integumentum: est genus demonstrationis sub fabulosa narratione veritatis involvens intellectum, unde dicitur etiam involucrum. Dieser Begriff dürfte auch nach Bernardus selbst den 'plot' der Cosmographia insgesamt bezeichnen. ${ }^{24}$ Für die in diesem 'plot' agierenden Gestalten selbst erscheint mir der Begriff 'Theophanie' am passendsten. ${ }^{25}$ Aus welchen Bestandteilen hat Bernardus innerhalb des integumentum seiner Cosmographia also seine Theophanie Silva zu einem lebendigen Charakter zusammengesetzt?

„Matter is really the heroine of the Cosmographia." ${ }^{26}$ Obwohl dieses dictum weder mit dem starken Zurücktreten der Silva im Microcosmus noch mit der Rolle

system, in which the integumenta were removed from nature and she was revealed to common view." Die allegorische Statuette „Die Natur enthüllt sich vor den Wissenschaften" des Franzosen Ernest Barrias (1905) verbildlicht so genau dieses Konzept (Abbildung bei Smolak [wie Anm. 18], Abb. 7), als ob Raby bei seinen Worten an sie gedacht hat.

${ }^{21}$ H.J. Westra (ed.), The Commentary on Martianus Capella's De Nuptiis Philologiae et Mercurii attributed to Bernardus Silvestris, Studies and Texts 80, Leiden 1986. Dronke (wie Anm. 6), 180, Anm. 1 spricht sich mit Jeauneau für die Authentizität des Kommentars aus, während Westra die Frage offen läßt.

${ }^{22}$ Ratkowitsch (wie Anm. 5), 22f. stellt die Schutzfunktion heraus, die in der Abkehr vom Traktat à la Wilhelm de Conches und in der Kreation eines kunstmythischen Prosimetrums bestanden haben könnte.

${ }^{23}$ J.W. \& E.F. Jones (edd.), The Commentary on the first six Books of the Aeneid of Virgil commonly attributed to Bernardus Silvestris, Lincoln / London 1977. Während sich Westra (wie Anm. 21), 8ff. für die Unsicherheit der Verfasserschaft beider Kommentare ausspricht, halten Jones / Jones die Verfasser beider Kommentare mit Bestimmtheit für identisch (X), beachten aber nicht den oben von mir herausgestellten Unterschied der Bestimmungen von allegoria, involucrum und integumentum. Sie weisen beide Kommentare in das Umfeld der Schule von Chartres (XII). Zu meinem bzw. (Ps.-?) Bernardus' Verständnis von allegoria vgl. Brinkmann (wie Anm. 6), 198, Anm. 909: „Allegoria ist für die christliche Deutung der heiligen Schrift reserviert.“

${ }^{24}$ Vgl. Donati (wie Anm. 7), 664 mit Anm. 44. Jüngst hat M. Camargo, A Twelfth-century Treatise on 'Dictamen' and Metaphor, Traditio 47, 1992, 161-213, hier: 165 die Möglichkeit eröffnet, daß Bernardus Silvestris einen Traktat über diese Phänomene geschrieben haben könnte.

${ }^{25}$ Nach Dronke (wie Anm. 18), 26 und Whitman (wie Anm. 6), 224. Sehr deutlich ist pace Pabst (wie Anm. 6), 454 der Unterschied zwischen den Theophanien des Bernardus und den allegorisch verstandenen herkömmlichen Gottesgestalten bei Martianus Capella.

${ }^{26}$ Stòck (wie Anm. 8), 232. 
der Natura überhaupt übereinstimmt, ${ }^{27}$ können wir zunächst doch feststellen, daß zumindest im Einleitungsmetrum die Handlung - und damit die Schöpfung! mittelbar von Silva ausgeht, deren Verlangen nach Gestaltung Natura dazu zu sollizitieren scheint, an Noys zu appellieren. Im Unterschied etwa zur Genesis fällt hier die starke Hervorhebung des Schöpfungssubstrats auf.

Wenn wir die Theophanie Silva im Megacosmus I betrachten, so stellen wir fest, daß hier bereits ${ }^{28}$ fast alle die Züge begegnen, die Silva durch die gesamte Cosmographia begleiten werden. Silva zeichnen folgende wesentliche Züge aus: $\mathrm{Ihr}$ Name 'Silva' ${ }^{\text {,29 }}$ kann durch Yle ersetzt werden (I 32 ante pedes assistit Yle cum prole suorum), was Bernardus wiederum synonym der Verbindung Usie vultus (I 19) behandelt. Diese Gleichsetzungen sind erklärungsbedürftig (1). Bestimmend für den Charakter der Silva ist die allgemeine Muttermetaphorik: I 32 wird von ihrer proles gesprochen, in I 39 wird sie regelrecht 'Mutter' genannt (Silva parens), als die sie einen 'Schoß' besitzt (gremium I 38), sie ist alumpna (I 42), die infantia Mundi befindet sich noch inter cunas (39), Mundus sehnt sich nach seinem natale (65). Wir fragen vor allem nach der Quelle dieser Muttermetaphorik (2). Bernardus legt großes Gewicht auf die informitas Silvae und ihre carentia. ${ }^{30}$ Diese beiden Prädikate sind zweideutig und oszillieren zwischen Nichtbesitz, Ungeformtheit und Armut, Häßlichkeit, besitzen also eine abstrakt-philosophische und eine personalmetaphorisch-emotionale Komponente. Hier drängt sich die Frage nach den Quellen des Bernardus und seinem Verhältnis $\mathrm{zu}$ ihnen auf (3). Integraler Bestandteil der silvestrischen Silva ist ferner ihre malitia, die in I 31 auctorem terrere und I 33 invidiam factura tibi anzuklingen scheint, aber erst in II 2 durch Noys als Hindernis der Gestaltung Silvas expliziert wird. Die malitia Silvae ist Chaos: Besonders zahlreich sind daher die Prädikationen der Silva, die ihren ungeordneten Zustand beschreiben, der durch Finsternis (I 29 si noctis habundat), ungeordnete materielle Häufung (I 1 congeries, I 2 sub veteri confusa globo u. ö.), inneren Kampf (I 12 miseros tumultus, I 18 concretio pugnax u. ö.) beschrieben wird und in der Bezeichnung 'chaos' kulminiert (I 18). Hier zeigen sich bemerkenswerte Parallelen zu topischen Chaosbeschreibungen in kosmogonischen Kontexten heidnischer Dichtung (4). Auffällig ist für den Leser der Genesis ferner der feste Wille der Silva, diesen Zustand zu verlassen, ${ }^{31}$ der ja allererst die Kosmogonie der Cosmographia initiiert. Woher hat Bernardus diese Aktivität der

${ }^{27}$ Pabst (wie Anm. 6), 448 bemerkt zutreffend, daß der Rezipient den Schöpfungsprozeß der Cosmographia durch die Augen der Natura erlebe.

${ }^{28}$ Mit Ausnahme des malitia-Konzepts, das erst in II 2 eindeutig eingeführt wird.

${ }^{29}$ I 1 , 8, 18, 29, 36, 37, 42, 59, 52, 57, 58 und die ganze Cosmographia hindurch.

${ }^{30}$ Informitas: I 1 informis, 18 informe chaos, 20 formam rudis, 31 male condita vultus, 34 informi squalore, 56 informi nudaque aula. Carentia: I 19 discolor, 29 lucis eget, 52 (pax, amor, lex, ordo) omnibus hiis quia Silva caret, 58 ut quid ab eterno comitata Carentia Silvam?

${ }^{31}$ I 21 optat, cupiens; I 22 requirit; I 32f. assistit [...] factura; I 35 cupit; I 40 orat. 
Silva (5)? Schließlich gehe ich kurz auf das Problem der Geschöpflichkeit der Silva und deren zeitlichen Aspekt ein, der für die Beurteilung des Bernardus vom theologisch-dogmatischen Standpunkt aus nicht ohne Bedeutung ist (6).

\section{DIE NAMEN DER SILVA}

Die Gleichsetzung von 'silva' und 'yle' als der auditiven, gräzisierenden Schreibung des aristotelischen Begriffs ' Übersetzung des griechischen Wortes in das lateinische Äquivalent der Alltags-, nicht der Philosophensprache. Man würde 'materia' erwarten. Zweifellos kommt der Begriff 'silva' den dramatischen Bedürfnissen des Bernardus entgegen, indem er einerseits nicht die technischen Assoziationen von 'materia' aufweist, andererseits aber eher nach einem Frauennamen klingt und daher die Mutter- und Ammenmetaphorik begünstigt.

Mustern wir flüchtig die Übersetzungsgeschichte von ' Berücksichtigung der Schriften, die Bernardus wahrscheinlich gekannt hat, so fällt auf, daß in der spätlateinischen philosophischen oder grammatischen Literatur der Begriff 'v̋ $\lambda \eta$ ' in der Regel glossiert begegnet: Die hermetische Schrift Asclepius, die Bernardus gekannt haben muß, ${ }^{32}$ spricht von $0 ँ \lambda \eta$ quem Graece credimus mundum. ${ }^{33}$ Wie bereits Gilson richtig erkannt hat, entstammt das silvestrische Vokabular am wahrscheinlichsten dem Timaios-Kommentar des Calcidius, aus dem allein die gesamte Silva-Konzeption des Bernardus stammen könnte. Calcidius gibt an zwei Stellen eine explizite Gleichsetzung von 'silva' und 'hyle' bzw. 'silva', 'hyle' und 'chaos', ${ }^{34}$ jeweils mit der Bemerkung, 'hyle' sei der griechische Begriff. Ebenso auffällig ist die wörtliche Parallele, die sich bei dem notorisch allegorisierenden Servius findet (ed. Rand zu Aeneis 1, 314): MEDIA SESE TULIT

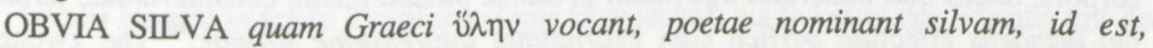

${ }^{32}$ Dafür sprechen vor allem die Begriffe 'Oyarses' (iii 2; v 5, 8, 13; vii 2), der eindeutig aus Asclepius III 19b, 27c (eine Liste von ov́orópxol, unter denen sich auch Zeus Hypatos, Pantomorphos, Imarmene, Zeus Nestos und Zeus Chthonios befinden) übernommen ist, und 'Pantomorphos' (iii 95-100), der auf Asclepius III 19b und III 35 (Scott 324. 10-12; 238. 26-29) zurückgeht. Vgl. dazu R.B. Woolsey, Bernard Silvester and the Hermetic Asclepius, Traditio 6, 1949, 340-44, hier: 342f., der 341 die mittelalterliche Verbreitung dieser synkretistischen Schrift des Corpus Hermeticum bei Roger Bacon, Daniel of Morley, Albertus Magnus und William of Auvergne belegt. Meiner Ansicht nach geht aus dem Asclepius-Zitat, das Thierry de Chartres in seinem Tractatus de sex dierum operibus zitiert, eindeutig hervor, daß der Asclepius sogar Bibliotheksbestandteil in Chartres war (Thierry ed. Häring 193. 26-27 = Asclepius I 310 und 314 Scott).

${ }^{33}$ Asclepius III 14b (310.26 Scott): fuit deus et quem Graece credimus mundum [...].

${ }^{34} 123$ (167 Waszink): chaos, quam Graeci hylen, nos silvam vocamus [...]; 248 (273 Waszink): necessitatem porro nunc appellat hylen, quam nos Latine silvam possumus nominare [...]. Dazu Gilson (wie Anm. 3), 10: „silva et hyle sont charactéristiques de l'influence de Chalcidius. B. Silvestris use de chaos, silva et hyle comme de trois synonymes." Präzisierend muß man allerdings feststellen, daß der bloße Gebrauch von derselben Sache mehrere Wörter noch lange nicht zu Synonymen macht. 
elementorum congeriem, unde cuncta procreantur. Hier konnte Bernardus ebenso gut alles Nötige finden: den terminus technicus der griechischen Philosophie, die Metonymie der lateinischen Dichtersprache und schließlich die philosophische Substanz beider Begriffe.

Trotzdem ist ein direkter Zugriff des Bernardus auf die beiden genannten Quellen nicht nachzuweisen, da die Gleichsetzung von 'chaos', 'hyle' und 'silva' in der Kommentartradition des zwölften Jahrhunderts bereits locus communis ist: So bemerkt ein anonymer, aber in der Substanz den Chartrensern nahestehender Kommentar zur Consolatio Philosophiae zum Substrat der Schöpfung Gottes: [...] illud chaos, quod olim erat, scilicet elementorum confusio, quae hyle appellatur, ${ }^{35}$ bei den namentlich identifizierbaren Chartrensern selbst begegnen folgende Gleichsetzungen: yle materia primordialis est; [...] materia primordialis quam alii ylem alii silvam alii cahos alii infernum [...] dixerunt mit deutlicher Beeinflussung durch Calcidius: ylen id est informis materia. ${ }^{36}$ Zusammenfassend: Die silvestrische Gleichung von Silva und Yle erscheint als eine mechanische Übernahme aus der zeitgenössischen Philosophie, die vermutlich darin vom lateinischen Neuplatonismus des Calcidius angeregt ist.

Die Bezeichnung discolor Usie vultus dagegen läßt sich nicht so glatt ableiten. Zwar liegen für den wiederum auditiv geschriebenen aristotelischen Begriff

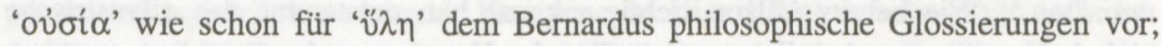
bei Augustinus kann er die Erklärung (scil.: essentia) quod Graeci appellant ov̉ $\sigma i \alpha v$ finden, ${ }^{37}$ ebenso in zeitgenössischen Kommentaren wie bei Gilbertus Porreta $\mathrm{zu}$ Boethius' De Trinitate. $^{38}$ Der Begriff muß in der zeitgenössischen Trinitätsdiskussion allgemein gebräuchlich gewesen $\operatorname{sein}^{39}$ Aus Calcidius wußte Bernardus, da $\beta$ es drei Semantiken von 'essentia' gebe, una quae constat ex corpore, altera qua materiam informem et adhuc silvam mente consideramus, tertia cui accidens effectum perficit eam exornatque impressione formae. ${ }^{40}$ Die zweite scheidet trotz der wörtlichen Parallele zwischen Bernardus und Calcidius ebenso aus, da Silva nur vultus Usie nicht Usia selbst sein soll. Der Meinung Silversteins,

${ }^{35}$ E.T. Silk (ed.), Saeculi noni auctoris in Boetii consolationem philosophiae commentarius, Papers and Monographs of the American Academy in Rome 9, Rome 1935, 157 im Kontext einer Erörterung des platonischen Dualismus zu Boethius Consolatio 3 m. 9. Die alte Datierung wurde durch Silk überzeugend ins zwölfte Jahrhundert korrigiert. Vgl. auch Dronke (wie Anm. 8), 181.

36 Thierry ed. Häring 275. 98; 272. 15; 172. 25.

${ }^{37}$ Civitas dei 12. 2 (edd. Dombart / Kalb 357. 7-16).

38 Gilberti Porretae Commentaria in librum De Trinitate Boethii, Migne PL 64. 1268. A 8f.: essentia quae Graece ov่oi $\alpha$ dicitur.

${ }^{39}$ Vgl. z.B. Hildebert de Lavardin (1056-1133), dessen Gedichte sehr verbreitet waren: in hoc deo, Deo vero / tres et unum assevero / dans usie unitatem / et personis trinitatem (c. 55: De sancta Trinitate ed. Scott, 80-84).

${ }^{40} 222$ (235f. Waszink). 
der die Äquivalenz von Silva und Usia an dieser Stelle annimmt, kann ich mich nicht anschließen. ${ }^{41}$ Räumt man die Möglichkeit ein, daß Bernardus 'Usia' in der Übersetzung Augustins für die erste calcidische essentia gebraucht haben könnte, so ergibt sich als plausibelste Lösung, daß der Ausdruck ungefähr bedeutet 'unfarbige Andeutung der körperlichen Dinge', die ja erst aus Silva hervorgehen werden. Vultus wäre dann als 'Oberfläche, oberflächliche Erscheinung' aufzufassen. Es wäre also nicht zu kühn, Silva als Andeutung der Körperwelt zu bezeichnen, als reine Potentialität. ${ }^{42}$ Wir werden in 3. darauf zurückkommen. Die andere Möglichkeit wäre, vultus wie 'imago' als 'Ebenbild' zu verstehen, das biblische Dogma von der Gottebenbildlichkeit des Menschen in kühner Verallgemeinerung auf das Schöpfungssubstrat zu verlagern und Usia dann im Sinne der dritten der calcidischen Möglichkeiten zu interpretieren: Wenn er den Begriff mit Kapitale schreibt, muß Dronke ihn als Namen einer Person verstanden haben. Bernardus verwendet in der gesamten Cosmographia aber alle drei der von Calcidius so mundgerecht differenzierten Bedeutungen: xii 17 und xiii 1. 4 eher im Sinne der ersten, II 2 und xiii 9 eher im Sinne der zweiten, II 1 im Sinne der dritten. Allerdings qualifiziert, wenn Bernardus die dritte Möglichkeit meint, meist noch ein Zusatz wie prima oder primeva die Usia. Eine solche Qualifikation findet sich in unserem Kontext aber nicht. Daher liegt es näher, daß Dronke Bernardus mißverstanden hat: usia sollte besser nicht als Name behandelt werden.

\section{MUTTERMETAPHORIK}

Bei der oben (134) nachgewiesenen Mutter- und Ammenmetaphorik kann sich Bernardus den Gleichklang von 'mater' und 'materia' zunutze gemacht haben, als die Silva auch verstanden werden kann (Summa Operis 3). Die materia prima wird bereits im platonischen Timaios in ihrer Eigenschaft als Substrat der Formen mit der Bezeichnung vं $\pi \circ \delta \circ \chi \eta \dot{~(51 ~ A ~ 5) ~ b e l e g t, ~ w a s ~ C a l c i d i u s ~ a n g e m e s s e n ~ a b s t r a k t ~ m i t ~}$ receptaculum übersetzt, dann aber in der letztlich hesiodeisch-homerischen Tradition auch mit zahlreichen Personalmetaphern versehen, die Calcidius an das Mittelalter weitergibt: Quae quidem corpora cum sola et per se ac sine suscipiente eadem essentia (hier also noch abstrakt) esse non possunt, quam modo matrem, alias nutriculam, interdum totius generationis gremium, nonnumquam locum appellat, quamque iuniores hylen, nos silvam vocamus. ${ }^{43}$ Da Bernardus diese Stelle sicher

41 Silverstein (wie Anm. 3), 99: „The further equivalence of usia for silva is not Chalcidean, but John the Scot had equated usia with essentia; and essentia, in turn, for Chalcidius is synonymous with silva."

42 Bernardus verwendet die dritte calcidische Möglichkeit in IV 4: Usya primeva. Für die Annahme des Ausdrucks vultus als 'Oberfläche, Andeutung, Potentialität' spräche II 4 (mit Kleinschreibung): erat Yle nature vultus antiquissimus, gleich darauf mit formarum prima subiectio materia corporum.

${ }^{43} 273$ Waszink 27. Vgl. auch 309, 9-13 Waszink. 
gekannt hat, war ihm der Ansatz seiner Muttermetaphorik bereits vorgegeben. $\mathrm{Zu}$ seiner Zeit war diese Parentalisierung der Hyle übliche Kommentarpraxis, wie folgende Zeilen eines anonymen Timaios-Kommentars, ebenfalls des zwölften Jahrhunderts, beweisen: erit igitur hyle quasi pater, secundum hoc quod assumptis formis transit in corpus, quasi mater secundum quod in se recipit formas et secundum quod pater principalis causa erit corporum, post deum et ideas, secundum quod mater secundaria causa. ${ }^{44} \mathrm{Da}$ Platon die $u ̈ \lambda \eta$ insgesamt mit einer Mutter vergleicht, begegnet uns auch im Bernardus zugeschriebenen Kommentar zu Martianus Capella: Plato in tractatu de yle sapientiam confert patri, ylem matri, mundum proli. Wenn wir sapientia durch die dem griechischen vov̂s zugehörige Theophanie des Bernardus, Noys, ersetzen, begegnen wir in diesem kurzen Satz bereits einem großen Teil des allegorischen Personals der Cosmographia. Wenn also Bernardus die philosophische Konzeption der $\ddot{u} \lambda \eta$ mit Personalmetaphern gewissermaßen bekleidet hat, so handelt es sich um eine folgerichtige Verlängerung der in der Kommentartradition zum Timaios längst metaphorisch angelegten Tendenzen zum Kunstmythos. Die Personalisierung wird in einem Bereich, in dem die Personalmetaphern zwar auch von der Tradition vorgegeben, aber deutlich als Vergleiche gekennzeichnet waren, erheblich deutlicher.

\section{INFORMITAS UND CARENTIA}

Die Klage der Natura vor Noys über den Zustand der Silva richtet sich in erster Linie auf deren informitas und carentia (vgl. oben 134 mit Anm. 30). Auch der Wille der Silva zur Veränderung beruht auf dem Abscheu vor ihrer forma rudis (I 20). Informitas und carentia bilden also im dramatischen Sinne die Handlungsmotivation.

'Informis' und 'carens' meinen in unserem Kontext dasselbe, da mit 'carens' natürlich 'carens forma' gemeint sein muß. Beide Begriffe oszillieren zwischen einem philosophischen Gehalt (kontradiktorisch: in-formis $=$ ohne Form, carens $=$ nicht besitzend) und einem emotional zu bewertenden ästhetischen (konträr: informis $=$ häßlich, carens $=$ armselig). Diese beiden Begriffsebenen sind bereits von spätantiken Grammatikern bemerkt worden. ${ }^{45}$ Priscian läßt im menschlichen Bereich ausdrücklich nur die ästhetische Bedeutung gelten, was den Schluß nahelegt, ihm sei auch die philosophische Verwendung bekannt gewesen. Daß Bernardus Silva im Urzustand als informis bezeichnet, beruht natürlich in erster Linie auf dem philosophisch-theologischen Bild, das man sich von dem Substrat der Schöpfung machte, erhält aber daneben unerwartete Unterstützung aus der

${ }^{44}$ Dronke (wie Anm. 6), 89 zitiert den Text aus dem bislang unveröffentlichten TimaiosKommentar des CLM 540 B (fol. 33r-v).

${ }^{45}$ ThLL s. v. informis $(1474,83 \mathrm{ff}$.): Prisc. gramm.: informis dicitur mulier non quae caret forma, sed quae male est formata; Gloss.: turpis [...] seu sine forma. 
antiken Synonymik. Um die Synonyme nemus, lucus und silva zu unterscheiden, behauptet Servius, daß Vergil die Synonyme vertausche, und fährt fort: interest autem inter nemus et silvam et lucum: lucus enim est arborum multitudo cum religione, nemus vero composita multitudo arborum, silva diffusa et inculta. ${ }^{46}$ Möglicherweise besitzt also silva assoziativ ohnehin schon den Beigeschmack der Dürftigkeit und beklagenswerten Armseligkeit. Wenn wir versuchen, eine oberflächliche Begriffsgeschichte der philosophischen Verwendungen von informis oder carens zu geben (griechische Äquivalente sollen uns hier nicht beschäftigen), so scheint der älteste Beleg im Apuleius platonicus aufzutauchen: Er spricht an zwei Stellen von materia inabsoluta, informis, nulla specie [...] distincta bzw. von materia rudis [...] qualitate viduata. ${ }^{47}$ Der Gedanke von der Urmaterie als einer nur durch die Subtraktion der praedicamenta zu bestimmenden Substanz beherrscht die gesamte Begriffsgeschichte in einer wiederum durch Calcidius vermittelten, letztlich aber aristotelischen Begrifflichkeit: ${ }^{48}$ Locus classicus ist das umfangreiche PhysikZitat, ${ }^{49}$ in dem Aristoteles bzw. Calcidius darlegen, daß die initia rerum drei seien (Waszink 289. 12ff.): silva, die als mater corporum formationi adiumentum (Waszink 290. 3ff.) fungiere, carentia, von der festgestellt wird: vero non adiuvat formationem, sed potius impedit ac renititur, schließlich species qua divina res et appetibilis. Nach einer formallogischen Erörterung dieser Beziehungen bleibt allein übrig, daß silva speciem cupit, um carentia zu beenden: sola ergo silva est quae cupit illustrationem, perinde ut femineus sexus virilem et deformitas pulchritudinem [...] (Waszink 291. 13f.). In nuce erkennen wir hier den Handlungskern der Eingangssituation unserer Cosmographia wieder, in dem allerdings die Aktivität der aristotelischen bzw. calcidischen silva auf die Theophanie der Natura verlagert wurde. Es bleibt festzuhalten, daß auch den Philosophen nichts anderes übrig

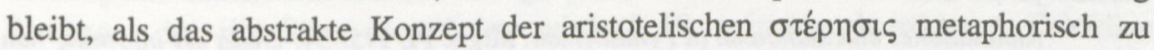
illustrieren. Und dies geschieht in genau der ästhetisch-personalen Ebene (femineus sexus bzw. deformitas), die uns in der Cosmographia begegnet. Eine Substanz, die aller Prädikate und damit jeder Anschaulichkeit beraubt ist, läßt sich natürlich ohnehin nur als reine materia inintelligibilis denken, weshalb im Grunde von silva informis überhaupt nur metaphorisch gesprochen werden kann: Fast notwendig folgt die Transposition von informis auf die ästhetische Ebene. Augustinus hat in seinen Confessiones diesen gedanklichen Fehler psychologisch einfühlsam wie immer beschrieben. ${ }^{50}$ Bernardus spricht gerade im philosophisch unzulässigen

${ }^{46} \mathrm{Zu}$ Aeneis 1, 310. Unter Umständen ist allerdings bereits diese Differenzierung des Servius von zeitgenössischen philosophischen Prädikationen der Urmaterie als silva beeinflußt.

${ }^{47}$ De Platone et eius Dogmate 1, 5.

48 In eine ähnliche Richtung denkt Stock (wie Anm. 8), 114: „Throughout the Cosmographia matter's need for formal ammelioration is presented in Aristotelian language."

49 Aristoteles Phys. I 9, 192a3-34 = Calcidius Comm. 286 Waszink 289f.

so 12.6 Skutella: foedas et horribiles formas perturbatis ordinibus volvebat animus, sed formas tamen et informe appellabam non quod careret forma, sed quod talem haberet, ut, si 
Sinne von informis. Sein genialer Schachzug besteht darin, den philosophischen Fehler der Anschaulichkeit für die Lebendigkeit seiner Allegorie auszunützen. Aufgrund der beschriebenen Quellen wird die Annahme einer materia informis qua privatione formarum in der philosophischen Tradition vor und um Bernardus Gemeingut: Johannes Scotus Eriugena ${ }^{51}$ faßt den Begriff noch einmal zusammen, Hugo de St. Victore, ${ }^{52}$ Thierry de Chartres ${ }^{53}$ und Alanus ab Insulis ${ }^{54}$ modifizieren ihn geringfügig. Betrachten wir das Verhältnis des Bernardus in seiner SilvaKonzeption $\mathrm{zu}$ diesen Zeugnissen, so ist evident, daß er sich für die nichtphilosophische, d. h. ästhetische Auffassung der Begriffe informis und carens entscheidet, wobei er sehr wohl durch Augustinus zu seiner Allegorese des philosophischen Begriffs von 'silva' gekommen sein könnte: Der Anschaulichkeit halber versetzt er sich in den philosophischen Anfänger zurück. Der Sinn des letztlich aristotelischen Prinzips, alle Kategorien zu verneinen, um zur reinen Potentialität zu gelangen, eben zur $\tilde{\nu} \lambda \eta$, wird bei ihm zur Verneinung bloß ästhetischer Kategorien: Silva ist nicht ohne Formen, sondern häßlich. ${ }^{55}$ Dabei bedient er sich zwar des philosophischen Vokabulars, nützt aber dessen immanente Ambiguität, um seine Allegorie mit ästhetischen Assoziationen zu bekleiden. Besonders pikant daran erscheint, daß die Philosophen ihr Vokabular aus einer

appareret, insolitum et incongruum aversaretur sensus meus et conturbaretur infirmitas hominis; verum autem illud quod cogitabam non privatione omnis formae, sed comparatione formosiorum erat informe, et suadebat vera ratio, ut omnis formae qualescumque reliquias omnino detraherem, si vellem prorsus informe cogitare et non poteram. Ebenso De Genesi ad Litteram Migne PL 34, 249-50 mit überraschender Parallele zu Boethius Consolatio 1 m. 2. 27.

${ }^{51}$ Periphyseon 1, $500 \mathrm{~B}(168,3 \mathrm{ff} . \mathrm{SW})$ : materia vero informis vocatur per privationum omnium formarum.

52 Annotationes elucidationum in Pentatichum: Migne PL 175. 34: creata est informis, non ex toto carens forma; sed ad conparationem sequentis pulchritudinis et ordinis, informis potest dici. Aus der Not der mangelnden Anschaulichkeit versucht Hugo also einen Mittelweg zwischen abstrakter und ästhetischer Bedeutung.

53 Thierry, ed. Häring (AHDLMA 30, 1955, 192), entwickelt aus der informitas seine These der uniformitas: informitas autem illorum elementorum in eo tunc consistebat quod unumquodque eorum fere erat huiusmodi quale alterum. ea quia minimum erat vel fere nihil quod intererat, idcirco illa differentia pro nihilo a philosophis reputabatur et illa [...] sic confusa una informis materia dicebatur. Nach A. Stollenberg, Der Genesiskommentar Thierrys von Chartres und die Thierry von Chartres zugeschriebenen Kommentare zu Boethius De Trinitate, Diss. Köln 1971, 43, Anm. 284 meine Thierry „frühmittelalterliche Philosophen, die im Anschluß an den Genesisbericht die mythischen Materiedarstellungen des Timaios und Augustins Ausführungen über die Materie (Aug. De gen. contra Man. 1, 5, 9; PL 34, 178) ihren Materiebegriff entwickelten."In diesem Punkt also scheint Bernardus nicht von Thierry abhängig zu sein.

${ }^{54}$ Sermo de Spera intelligibilis (ed. D'Alverny), 299: primordialis vero materia que orbiculari formarum circumfertur et ymaginationis fantasia concipitur merito spera ymaginibilis esse censetur - ein regelrechtes Motto für die Silva-Allegorese des Bernardus.

ss Ähnlich H. Flatten, Die Materia primordialis in der Schule von Chartres, AGPh 40, 1931, 58-65, hier: 63: „In der Lehre Bernhards hat die materia prima viel von ihrem aristotelischen Sinn eingebüßt [...]. Wenn man genauer hinsieht, zeigt sich, daß die negative Darstellung nicht im metaphysischen Sinne die Aktualität zugunsten der Potentialität verneinen soll, sondern nur die rohe Urmaterie gegenüber der materia formata als minderwertig bezeichnet." 
gewissen sprachlichen Impotenz heraus ebenfalls nur bildlich verwenden: Die Beispiele, die Aristoteles bei Calcidius bringt (femineus sexus, deformitas), werden bei Bernardus im integumentum zu proprie gebrauchten, d.h. ästhetischemotionalen Beschreibungen der Silva. Dabei kümmert er sich nicht um die Stimmigkeit seiner Übertragung: Bei Aristoteles bzw. Calcidius war carentia neben silva und species ein selbständiges Prinzip, bei Bernardus ist sie plötzlich ein Modus der Silva geworden, der personifiziert von Natura weggewünscht wird (I 58). ${ }^{56}$ In der philosophischen Tradition ist informitas die substantia der materia primordialis, bei Bernardus wird dieselbe informitas ins Ästhetische allegorisiert und somit zu einem bedauerlichen Akzidens, um dessen Beseitigung die Cosmographia kreist. Weiterhin ist die Transformation ursprünglich philosophischer über ästhetische schließlich zu moralischen Kategorien für die allegorische Technik des Bernardus kennzeichnend: Die malitia Silvae (II 2, vgl. Anm. 28), auf die hier nicht weiter eingegangen werden soll, ${ }^{57}$ bezieht ihre suggestive Folgerichtigkeit aus der Empfindung der Natura, die den häßlichen Zustand als Schlechtigkeit versteht. Diese Schlechtigkeit läßt sich mit dem essentiell guten Wesen Gottes nicht vereinbaren und muß deshalb beseitigt werden. Man erkennt, wie frei Bernardus mit der philosophischen Tradition schaltet, die er gerade so weit verändert, daß zwar etwas Neues entsteht, die zugrundeliegenden Konzepte aber noch sichtbar bleiben.

\section{TOPIK IN DER BESCHREIBUNG DES URCHAOS}

Bisher haben nahezu ausschließlich philosophische Quellen eine Rolle in der Konzeption der silvestrischen Silva gespielt. Obwohl auch die Beschreibung des Urchaos, das mit Silva identifiziert wird (I 18 informe chaos), aus Calcidius abgeleitet werden könnte, lassen sich auffälligere Parallelen $\mathrm{zu}$ dichterischen Chaosbeschreibungen der klassischen römischen Literatur ziehen, die sich insgesamt derartig ähneln, daß man von einer festen Topik sprechen kann. Feste Bestandteile dieser Topik sind die Erwähnung der Anfangszeit ([1] - bei Bernardus: I 2 primordia rerum, I 21 vetus tumultus, I 35 res antiquissima), der indistinkte Mischungszustand der zukünftigen Weltbestandteile ([2] - bei Bernardus: I 1 congeries, 29 nox, 48 permixta trahit confusus acervus u. a.), der Kampf der Elemente ([3] - bei Bernardus: I 18 concretio pugnax, I 19 sibi dissona massa, I 24 ingenite lites, I 25 sibi contraria moles).

${ }^{56}$ Die Berechtigung, carentia in I 58 als Personifikation aufzufassen, kann angezweifelt werden, da sie in der Cosmographia nie wieder als Person auftritt und comitata natürlich auch buchstäblich verstanden werden kann.

57 Ratkowitsch (wie Anm. 5), 29-32 legt den malitia-Gedanken ihrem Theodizee-Konzept zugrunde. 
In der Ars Amatoria Ovids und dessen Metamorphosen konnte Bernardus die Elemente [1] und [2] finden. ${ }^{58}$ Aufgrund wörtlicher Parallelen, die nicht direkt zur Chaostopik gehören, ist hier ein direkter Einfluß anzunehmen: ${ }^{59}$ Ovids Begriffe vultus naturae (V. 6) und melior natura (V. 21) haben offensichtlich anregend auf die Formulierungen Usie vultus (19) und melioris forme (9) eingewirkt. Bernardus will den Leser also an das Proömium der Metamorphosen erinnern. Der Sinn dieser Anspielungen könnte sein, daß Bernardus seine Cosmographia sogar als 'Metamorphose' verstanden wissen möchte, als die Grundmetamorphose, die allen anderen Metamorphosen zugrundeliegt: Das ungestalte Etwas wandelt sich in die Schöpfung. Falls Bernardus Manilius gekannt hat, hätte er sich an die Verse 1, 125f. der Astronomica erinnern können. ${ }^{60}$ Lucan verwendet in seinem Bürgerkriegsepos ebenfalls die Motive [1] und [2]. ${ }^{61}$ Einer Andeutung des Martianus Capella in De Nuptiis konnte er neben anderen Quellen den dritten Bestandteil der Chaostopik entnehmen, den Institutiones divinae des Laktanz wiederum [1] und [2]. ${ }^{62}$ Boethius schließlich betont Motiv [3] in seinem Preis des kosmischen amor in Consolatio $2 \mathrm{~m} .8$ und $4 \mathrm{~m} .6^{63}$ Dieses Material war jedem Literaten des zwölften Jahrhunderts mühelos verfügbar. In der unmittelbaren Umgebung des Bernardus bedienen sich Wilhelm de Conches und Thierry de Chartres einiger Ausdrücke über den Anfangszustand, die an die Cosmographia denken lassen. In den Versen I $43 \mathrm{ff}$. handelt Bernardus von der Spontanbewegung der Elemente im Urzustand, die er

${ }^{58}$ Ars 2, 467ff., Met. 1, 1-17.

59 Diese wörtlichen Parallelen schließen die andere Möglichkeit aus, daß beide unabhängig voneinander von einer ähnlichen Textgruppe zu ihren Formulierungen inspiriert sein könnten. In diesem Zusammenhang soll nicht unterschlagen werden, daß sogar eine Affinität zwischen der Genesis und dem Beginn der Metamorphosen erwogen wurde: Vgl. W. Speyer, Spuren der 'Genesis' in Ovids Metamorphosen?, in: U.J. Stache, W. Maaz, F. Wagner (Hrsgg.), Kontinuität und Wandel. Lateinische Poesie von Naevius bis Baudelaire. FS F. Munari, Hildesheim 1986, 9099. Sinnvoller erscheint es vielmehr, über die Verbindung von Hesiod, den 'orphischen' Kosmogonien und der Genesis nachzudenken, um Ovid dann aus der heidnischen Tradition abzuleiten.

${ }^{60}$ Seu permixta chaos rerum primordia quondam discrevit partu [...]. Hier begegnet der sonst nur noch bei Lukrez bezeugte Ausdruck primordia rerum $(1,55 ; 483)$, außerdem ebenfalls die Konzeption einer Diakrisis-Kosmogonie, deren Agens aber offensichtlich das Chaos selbst ist: drei Parallelen zu Bernardus! Nach der gängigen Meinung war Manilius im Mittelalter unbekannt (z.B. M. Manitius, Geschichte der lateinischen Literatur des Mittelalters, Dritter Teil unter Mitwirkung von P. Lehmann, München 1931, 2. 747, Anm. 2), gegen die allerdings D.B. Gain, Gerbert und Manilius, Latomus 29, 1970, 128-32 Einspruch erhebt.

${ }^{61} 1,73 \mathrm{f}$., wo die vom vorletzten Herausgeber diagnostizierte Interpolation geradezu das Bestehen einer Chaostopik in der Spätantike erweist, nach der man solche Interpolationen vornehmen konnte. Außerdem sticht in Vers 80 das wiederum lukrezische Signalwort machina ins Auge, das ebenfalls bei Bernardus begegnet (I 54, vi 26, x 52).

${ }^{62}$ Martianus Nupt. 1, 92 quaeque elementa liget dissona nexio. Lactanz Div. Inst. 1, 5, 4-14 passim, v. a. 8: (Homer fällt als Zeuge für den vorchristlichen Monotheismus aus, weil er nur Menschliches berichtet) potuit Hesiodus sed tamen nihil, dedit non a deo conditore sumens exordium, sed a chao, quod est rudis inordinataeque materiae confusa congeries.

${ }^{63}$ Cons. 2 m. 8.3f., 4 m. 6.19ff. 
nach dem platonischen Timaios in verschiedene Richtungen streben läßt. $\mathrm{Zu}$ eben diesem Thema bemerkt Wilhelm: ante exornationem erant elementa divisa sedibus. [...] non dixit 'commixtio' sed 'confusio'. non enim tunc erant elementa commixta, ut quidam putant, sed unumquodque separatum ab alio, sed confusum in eo, in hoc scilicet quod habebat ornatum suum. ${ }^{64}$ Bei Bernardus scheinen die Elemente aber confusa (I 38) zu sein, so daß er vielleicht gegen Wilhelm polemisiert. Das Signalwort congeries begegnet bei Thierry in der Bemerkung, daß Rauch eine congeries guttarum sei. ${ }^{65}$ Vielleicht war diese Vokabel im 'naturwissenschaftlichen' Jargon der Chartrenser sogar terminologisch. Immerhin scheint im zwölften Jahrhundert diese Chaostopik so gängig zu sein, daß man sich ihrer mühelos verfremdend zu bedienen vermag. ${ }^{66}$

Man wird abschließend nicht entscheiden können, ob Bernardus seine Chaosschilderungen als aemulatio Ovids verstanden hat, ob er rein assoziativ sich dieser Ausdrücke bedient oder ob sie zu seiner Zeit im philosophischen Vokabular bereits so gängig waren, daß die Anklänge schlicht unbeabsichtigt sind. Die Parallelität zwischen dichterischem und philosophischem Vokabular (etwa bei Calcidius passim) in der Beschreibung des chaotischen Urzustandes läßt sich am leichtesten durch die Übernahme letztlich hesiodeischer Gedanken in den platonischen Kunstmythos des Timaios erklären. Aus der Sicht des Bernardus aber lagen zwei parallele Traditionen vor: indirekte Hesiodrezeption über Ovid und indirekte Platonrezeption über Calcidius, für Bernardus einfach Ovid und Calcidius. Für den literarischen Eklektizismus der Cosmographia spricht es, daß Bernardus sich hier eher der dichterischen Tradition anschließt. Obwohl keine wörtlichen Anklänge zur Eingangssituation der Genesis vorliegen, ist doch bemerkenswert, daß sich das Chaos nur auf Silva bezieht, daß sie es sogar 'ist', während sich über diesem Chaos Noys und Natura in schönster, gewissermaßen supralunarer Ordnung begegnen. Denjenigen Punkt, in dem sich die Genesis von den klassischheidnischen Kosmogonie-Szenarien unterscheidet, nämlich die Polarität zwischen dem Geist Gottes und dem Chaos bzw. dem abyssus, weist, wenn man Noys und Natura als Theophanien, als personifizierte Extrapolationen bestimmter Wesenszüge Gottes begreift, auch die Cosmographia auf. Das ist ein Indiz dafür, daß die Genesis doch deutlicher Pate gestanden hat für die Cosmographia, als es zunächst den Anschein hatte. Ferner fällt auf, daß Bernardus im Gegensatz zur dichterischen Tradition das Chaos quasi naturwissenschaftlich begründet: An diesem Durcheinander sei das ständige Entstehen und Vergehen von naturae in dieser

${ }^{64}$ Glosae super Platonem 157, ed. Jeauneau 289.

${ }^{65}$ De sex dierum Operibus § 7, ed. Häring AHDLMA 30, 1955, 186.

${ }^{66}$ Das tut z. B. Walther v. Châtillon (?) in einem eigenartigen Klagegedicht auf den Zustand der ecclesia, dessen zweite Strophe lautet: Ab antiqua rerum congerie / cum pugnarent rudes materie / moles fuit huius elegiae / ordinata (zuerst ediert von A. Wilmart, Poèmes de Gautier de Châtillon dans un manuscrit de Charlesville, RB 49, 1937, 143-167). Das Gedicht stammt aus einer Sammlung des ausgehenden zwölften Jahrhunderts. Die Urheberschaft ist nicht gesichert. 
Mischung schuld. ${ }^{67}$ Auch für den Fall der Chaostopik ergibt sich ein ähnliches Bild wie bei der Beleuchtung der Begriffe carentia / informitas: Bernardus beherrscht die Tradition souverän genug, um herkömmliche Züge neu zu kontextualisieren.

\section{DER WILLE DER SILVA}

Bei den bisher behandelten Konzepten befand sich Bernardus nicht in dezidiertem Widerspruch zur christlichen Dogmatik. Seine Konzeptionen berührten sich nicht mit biblischen Texten. Der Wille der Silva aber, ausgestaltet zu werden, der den Ablauf der Cosmographia in Gang setzt (s. 130f.), verleiht der Silva ein Gewicht, das zu den in der Genesis berichteten Fakten in klarem Widerspruch steht: Dort war alles Folge des Gottesworts, in der Cosmographia aber muß Natura für Silva aktiv werden und Noys überzeugen. Letztlich geht also, wenn auch auf eine seltsam passive Weise, die Aktivität von Silva selbst aus. Dieses Konzept ist typisch calcidisch, findet sich aber überall dort, wo die Idee einer 'kosmischen Liebe' für die Weltgestaltung herangezogen wird. ${ }^{68}$ Das Verhalten der Silva ist durch den Wunsch bestimmt, a veteri [...] exire tumultu (I 21), sie kann dieses Verlangen aus eigener Kraft aber offenbar nicht befriedigen. Mit Sicherheit beruht diese cupiditas Silvae auf Calcidius, der in seiner Diskussion der Physik-Passage des Aristoteles deutlich hervorhebt, daß silva es sei, die es nach illustratio verlange wie den femineus sexus nach dem sexus virilis. ${ }^{69}$ Wie schon bei seiner Version der informitas und carentia silvae (oben 138-141) folgt Bernardus hier der Begrifflichkeit des Calcidius, mißachtet aber dessen Kautel negat esse talem qualis est amimalium (vgl. Anm. 69). Er gebraucht dabei die Begriffe proprie, die Calcidius metaphorisch verwendet, und erreicht so hohe Lebendigkeit und Anschaulichkeit, die die philosophischen Konzepte dahinter doch noch erkennen läßt. Dabei setzt er sich über die christliche Kritik an der Aktivität der Ursubstanz hinweg, für die hier als Beispiel nur Tertullian angeführt werden soll. Dieser versucht in Adversus Hermogenem 41f., dessen Konzept einer schöpfungsaktiven

${ }^{67}$ II 4 stabilitatem bonumque tranquillitatis excussit frequens nec intercisa frequentatio naturarum. egredientium numerus ingredientibus locum pandit.

${ }^{68}$ P. Dronke, L'Amor che move il Sole e l'altre Stelle, SM 3a s. 6, 1965, 389-422 verfolgt die Idee dieser kosmischen Liebe vom homerischen Aphrodite-Hymnus über die Tragiker, Lukrez, Apuleius, den Neuplatonismus, Boethius, die Chartrenser, Dante und Boccaccio bis hin zu Chaucer. Die Silva des Bernardus bettet er 414 in diese Entwicklung ein: „Yet the universe, insofar as it is unformed, longs for this love."

69 Calcidius Comm. 286 (Waszink 290ff.): quia obscurior sermo est, explanandus videtur. tres ab eo ponuntur origines universae rei, species silva carentia (Waszink 291.9ff.) [...] sola ergo silva est quae cupit illustrationem, perinde ut femineus sexus virilem et deformitas pulchritudinem, ita tamen ut deformitas silvae non ex natura sed ex accidentia sit (Waszink 291.13f.) [...] superest 'ergo, ut silva cultum ornatumque desideret, quae deformis est non ex se, sed ob indigentiam. Est enim turpitudo silvam cultu formaque indigere (Waszink 291.16). [...] cupiditatem vero negat esse talem, qualis est animalium, sed, ut cum quid coeptum atque inchoatum, dicitur perfectionem desiderare, sic opinor, etiam silva speciem cupit (Waszink 292.4). 
materia, das dem des Calcidius in diesem Punkt sehr ähnlich ist, durch den Aufweis innerer Widersprüche zu entkräften: Die informitas materiae verbiete es dieser, formam zu erstreben, da nichts sein eigenes Gegenteil erstreben könne, ohne zu vergehen. Es sei aber widersprüchlich, daß etwas nach seinem Untergang strebe. ${ }^{70}$ Mit demselben Argument bewiesen Aristoteles bzw. Calcidius daß nicht carentia, sondern silva speciem ersehnen müsse. ${ }^{11}$ Das Szenario des Megacosmus I widerspricht also implizit der christlichen Kritik an neuplatonischen Weltentstehungsmodellen und setzt sich dabei auch über solche Autoritäten wie Johannes Scotus Eriugena hinweg, der die Materie als vollkommen passiv gesehen hatte. $^{72}$

\section{DIE GESCHÖPFLICHKEIT DER SILVA}

Neben der Quisquilie, ob Silva nun den Schöpfungsvorgang aktiv auslöse oder sich ihm passiv unterwerfe, und den Implikationen der Antwort für die Einschätzung des Bernardus ist die Frage, ob Silva bei Bernardus überhaupt von Gott geschaffen sei oder eine Art Gegenprinzip zu den Theophanien Noys und Natura darstelle, wesentlich interessanter und daher auch öfter gestellt worden. Neuerdings war Lomperis immerhin so vorsichtig, sich die Antwort offenzuhalten, für ihre Unsicherheit aber Bernardus selbst verantwortlich $\mathrm{zu}$ machen. ${ }^{73}$ Weitverbreitet ist hingegen die Meinung, bei Silva und Gott in seinen Aspekten Noys und Natura handele es sich um ,deux principes irréductibles“, 74 weil die Schöpfung der Materie gar nicht erwähnt werde, so daß Gottes Schöpfungsleistung nur im Ordnen eines chaotischen Gegenprinzips bestehe. ${ }^{75}$ Die Formulierungen melioris formae und das Vergilzitat mollius excudi wurden in dem Sinne verstanden, daß der Komparativ einen Zustand, in dem Silva bereits existent sei und im aristotelischen Sinne aktuell

70 Tertullian Adv. Hermog. 42, 1: dispersisti omnia, ne de proximo quam contraria sibi sint reluceret. at ego colligam singula et conferam. inconditum adseveras motum materiae eamque adicis sectari informitatem; dehinc alibi, desiderare componi a deo.

${ }^{71}$ Calcidius Comm. 286 (Waszink 290ff.), vgl. Anm. 69.

72 Periphyseon 500B (SW 168.3-5): materia vero informis vocatur per privationem omnium formarum ab ea siquidem nihil formatur, sed diversas recipit formas. $500 \mathrm{D}$ (SW 168.20f.): mutabilitas rerum mutabilium capax omnium formarum materia est. Eriugena folgt damit der herkömmlichen Auffasssung der Materie als reiner Potentialität und verwendet zusätzlich die ebenfalls herkömmliche Muttermetaphorik.

${ }^{73}$ L. Lomperis, From God's Book to the Play in the Cosmographia, MH n.s. 16, 1988, 5171, hier: 53: ,[...] the Cosmographia seems to begin by making the paternity of the opus, and indeed of the opus itself, unclear." L. redet im Sinne ihrer (wohl haltlosen) Theorie von der bewußten Gleichsetzung zwischen Weltschöpfung und Dichtungsvorgang in der Cosmographia.

74 Gilson (wie Anm. 3), 20, der die Cosmographia für die christliche Deutung retten möchte, formuliert in dieser Weise die Ansicht, gegen die er argumentiert.

75 Ebd. 9. Dieser Sicht hat sich M.D. Chenu, La Theologie au Douzieme Siècle, Paris 1957, 114 angeschlossen. Ähnlich Gregory Anima (wie Anm. 4), $65 f$. 
vorliege ${ }^{76}$ als Vergleichspunkt voraussetze. Das letztlich aus dem platonischen $\delta \eta \mu$ iovprós-Gedanken stammende Argument, daß Gott doch einen Stoff zur Schöpfung benötige, wird allerdings bereits von Laktanz in Auseinandersetzung mit Cicero als der Allmacht Gottes widersprechend und daher massiv häretisch zurückgewiesen. ${ }^{77}$ Wenn Bernardus einen Urdualismus propagierte, wäre er tatsächlich als neopaganer oder manichäischer Häretiker einzuschätzen. Aber er sagt in Megacosmus I deutlich genug, wie Gott und Silva zueinander stehen: I 4 operique suo non derogat auctor, I 31 (si possit) auctorem terrere suo male condita vultu, I 63 fateatur opus quis fecerat auctor beweisen, daß Bernardus Gott als auctor seines opus, der Silva und des aus dieser hervorgehenden mundus, auffaßt. Es kann also von zwei irreduziblen Prinzipien nicht im mindesten die Rede sein: Die Existenz der Silva gründet eindeutig in Gott. Dieses Verhältnis gibt dem Appell Naturae ja auch seine Kraft, da der auctor für sein opus eine Verantwortung trägt. Die beiden Komparative müssen also nicht im Sinne eines ewigen Vorliegens der Silva vor ihrer Ausgestaltung verstanden werden, sondern als Hinweis auf eine Dreistadienlehre: Erst war nur Gott, dann schuf er eine materia informis, dann aus dieser den Kosmos. Eine solche Auslegung ermöglicht die Koexistenz der auctorBegrifflichkeit und der Annahme einer materia informis. Diese Ansicht scheint in der Genesis-Exegese üblich gewesen zu sein. ${ }^{78}$ Außerdem spricht Bernardus deutlich genug vom ortus Silvae. ${ }^{79}$ Daß Silva die antiquissima res (I 35) ist, ließe sich sogar dann mit der vorgetragenen Auffassung versöhnen, wenn man Gott als res betrachtete und den Superlativ nicht elativisch auffaßte, was beides eher unwahrscheinlich erscheint. Denn selbst wenn Gott res wäre, müßte seine Existenz doch überzeitlich sein,$^{80}$ so daß Silva antiquissima res in tempore sein könnte und trotzdem nicht als mit Gott gleichursprünglich zu verstehen wäre. In dieser könnte man die Cosmographia also als integumentum der Genesis verstehen, mit den begrifflichen Mitteln, die in der Exegese in Chartres gängig waren. ${ }^{81}$ Dieselben oder

${ }^{76}$ So Flatten (wie Anm. 55), 61.

77 Lactantius Div. Inst. 2.8.26: ut faber inquit [scil. Cicero] cum quid aedificaturus est, non ipse facit materiam, sed utitur ea quae sit parata, fictorque item cera, sic isti providentiae divinae materiam praesto esse oportuit, non quam ipsa faceret, sed quam haberet paratam. immo vero non oportuit: erit enim deus minoris potestatis, si ex parato facit, quod est hominis. faber sine ligno nihil aedificabit, quia lignum facere non potest.

78 Vgl. Augustinus Gen. Litt. 1.5, PL 34.250 Migne, ähnlich Hugo de St. Victore, Eluc. Ann. in Pent. 5, PL 175.34 Migne und Thierry de Chartres (ed. Häring AHDLMA 30, 1955, 186): in principio igitur creavit Deus caelum et terram, id est materiam in primo momento temporum creavit (5).

${ }^{79}$ I 28, I 36. Wenn Silva einen ortus novus will, muß es für Bernardus einen ortus antiquus gegeben haben.

${ }^{80}$ Schon der heidnische Neuplatonismus war von der Überzeitlichkeit Gottes ausgegangen: Vgl. z. B. Tiberianus, MLP 566.7-11 Duff. Boethius Cons. Phil. 5.6.7-8, 10-11, 14 und Augustinus Conf. 11.8, 13, 16; Civ. 11.6; 12.15 lassen keinen Zweifel an der Überzeitlichkeit des christlichen Gottes, der simultan alle Zeit erkennt und ist.

${ }^{81}$ Gilson (wie Anm. 3), 8 kommt anhand anderer Fragen zu einem ähnlichen Ergebnis. 
ähnliche Techniken in der Umformung von einer christlichen oder heidnischen Tradition entnommenen Allegorien zu belebten Charakteren mit eigenständigem Persönlichkeitsbild, die wir hier ansatzweise für die Silva des Bernardus dokumentiert haben, ließen sich ebenso gut für das restliche Personal der Cosmographia nachweisen. ${ }^{82}$ Bernardus demonstriert, welche Metamorphose ein naturwissenschaftlich-philosophischer Text wie der des Calcidius erlebt, wenn man ihn konsequent unter Ausnutzung seiner eigenen Metaphorik allegorisiert.

MARKUS ASPER

${ }^{82}$ Für Natura ist das schon häufig versucht worden: Vgl. den Überblick bei Ratkowitsch (wie Anm. 5), 39-45. 